\title{
Adherence and Efficacy of Smoking Cessation Treatment Among Patients with COPD in China
}

\author{
Rui Qin ${ }^{1-6}$ \\ Zhao Liu ${ }^{1,3-6}$ \\ Xinmei Zhou ${ }^{1,3-6}$ \\ Anqi Cheng ${ }^{1,3-6}$ \\ Ziyang Cui ${ }^{1,3-7}$ \\ Jinxuan $\mathrm{Li}^{1,3-6,8}$ \\ Xiaowen $\mathrm{Wei}^{1,3-6,8}$ \\ Dan Xiao ${ }^{1,3-6}$ \\ Chen Wang ${ }^{1,3-7}$
}

'Tobacco Medicine and Tobacco Cessation Centre, Center of Respiratory Medicine, China-Japan Friendship Hospital, Beijing, People's Republic of China; ${ }^{2}$ Peking University China-Japan Friendship School of Clinical Medicine, Beijing, People's Republic of China; ${ }^{3}$ National Center for Respiratory Medicine, Beijing, People's Republic of China; ${ }^{4}$ Institute of Respiratory Medicine, Chinese Academy of Medical Sciences, Beijing, People's Republic of China; ${ }^{5}$ National Clinical Research Center for Respiratory Diseases, Beijing, People's Republic of China; ' $\mathrm{WHO}$ Collaborating Centre for Tobacco Cessation and Respiratory Diseases Prevention, Beijing, People's Republic of China; ${ }^{7}$ Peking Union Medical College, Chinese Academy of Medical Sciences, Beijing, People's Republic of China; ${ }^{8}$ Capital Medical University, Beijing, People's Republic of China

Correspondence: Chen Wang

Peking Union Medical College, Chinese

Academy of Medical Sciences, No. 9,

Dongdan San Tiao, Dongcheng District,

Beijing, 100730, People's Republic of

China

Tel + 010-65I05565

Email wangchen@pumc.edu.cn

Dan Xiao

Tobacco Medicine and Tobacco Cessation Centre, Center of Respiratory Medicine, China-Japan Friendship Hospital, No. 2

Yinghua East Street, Chaoyang District,

Beijing, 100029, People's Republic of

China

Tel + 010-84205425

Email danxiao@263.net
Background: Smoking cessation is a key intervention for all smokers with chronic obstructive pulmonary disease (COPD). Poor treatment adherence is a challenge in clinical practice that might contribute to the lower efficacy of medication (eg, oral drug). However, it is unclear what factors will influence adherence among smokers with COPD.

Methods: This study was based on an open-label randomized controlled trial (RCT) of varenicline and bupropion for smoking cessation among patients with COPD in China. The medication was given for 12 weeks, and visits and assessments were conducted at weeks 0 , $1,2,4,6,9,12$, and 24 . We assessed whether the adherence to smoking cessation treatment affects the smoking cessation efficacy and evaluated predictors of adherence.

Results: A total of 136 participants were recruited from February 2019 to June 2020, and analyzed using the intention-to-treat (ITT) method. In this study, 48.5\% (66/136) of the total participants had good adherence to smoking cessation, and good adherence significantly improved the efficacy of smoking cessation $(\mathrm{OR}=9.60,95 \% \mathrm{CI} 4.02-22.96, \mathrm{P}<0.001)$. After adjusting for age, gender, nationality, education, and marital status, we found older age, higher education level, having more previous quitting attempts, stronger self-efficacy and preparation in quitting smoking, recognizing hazards of smoking, longer duration of COPD, and higher St. George's Respiratory Questionnaire (SGRQ) scores were relevant to good adherence $(\mathrm{P}<0.05)$.

Conclusion: To our best knowledge, this is the first study to evaluate adherence to smoking cessation treatment among patients with COPD in China. Our study found that good adherence to smoking cessation treatment significantly improved the smoking cessation efficacy, and predictors of adherence were evaluated. We call on the medical community to pay attention to the adherence to smoking cessation among patients with COPD.

Keywords: COPD, smoking cessation, adherence, China

\section{Introduction}

Chronic obstructive pulmonary disease (COPD) is a common respiratory disease characterized by persistent airflow limitations and respiratory symptoms. ${ }^{1}$ COPD is a worldwide public health challenge because of its high prevalence and disability, and it remains the third leading cause of death worldwide in $2016^{2}$

The economic and social burden of COPD in China is heavier than that in developed countries, with COPD rapidly becoming a leading cause of mortality in China. ${ }^{3,4}$ It is estimated that the overall prevalence of COPD in China is $8.6 \%$, accounting for 99.9 million in $2018 .^{4}$

The 2021 Global Initiative for Chronic Obstructive Lung Disease (GOLD) guideline points out that the risk for developing COPD results from an interaction 
between genetics and many environmental factors, and smoking is the most common environmental factor. For example, a higher prevalence of respiratory symptoms and mortality rates of COPD are seen among smokers than non-smokers. ${ }^{1}$

Smoking cessation is a key evidence-based intervention for all smokers with COPD, which can slow the accelerated decline in lung function and reduce the risk of development of COPD. ${ }^{1}$ To date, the treatment for smoking cessation mainly includes psychological intervention, behavioral support, pharmacotherapy, etc. ${ }^{5}$ Among them, pharmacotherapy can significantly improve the success rate of smoking cessation. ${ }^{6}$ Nevertheless, despite different interventions for smoking cessation, the chance of smokers with COPD definitively quitting smoking was still low in the real world. ${ }^{7}$

European Society for Patient Adherence, Compliance and Persistence defined medication/treatment adherence as "the process by which patients take their medication/treatment as prescribed". ${ }^{8}$ Poor treatment adherence is a challenge in clinical practice that might reduce the efficacy of medication (eg, oral drug). ${ }^{8}$ Previous studies founded that $20 \%$ of the smokers who receive pharmacotherapies for smoking cessation never take medication as prescribed, ${ }^{9,10}$ and smokers often use them in fewer doses and for less time than health professionals suggest. For instance, good adherence to smoking cessation treatment occurs among $50 \%$ or fewer users of nicotine replacement therapy. ${ }^{11}$

Previous studies related to smoking cessation treatment adherence mostly focused on the general population. However, most studies did not distinguish different kinds of smokers, although there is some evidence that smokingrelated features differ between smokers with COPD and smokers without COPD. For example, smokers with COPD might have more difficulty quitting smoking because of their longer duration of smoking, and lower self-efficacy. ${ }^{12}$ This suggests that smokers maybe are not a homogeneous population, and therefore it is important to make the intervention most suitable for smokers with COPD. $^{13}$

Moreover, predictors of adherence to smoking cessation treatment may vary based on the population being assessed. Individuals with chronic diseases (eg, COPD) may have more difficulties as the use of smoking cessation medication further complicates medication self-management, and they tend to face a greater burden of smoking-related morbidity compared with the general population. ${ }^{11,14}$ Therefore, identified predictors of adherence maybe not be generalized to patients with chronic diseases, and this highlights the need for more studies to evaluate predictors of adherence for these diseases.

There are a few studies about adherence to smoking cessation treatment among patients with chronic diseases. ${ }^{11,15}$ However, to the best of our knowledge, it is unclear what factors will influence adherence to smoking cessation among smokers with COPD.

Therefore, this study aimed to assess whether the adherence to smoking cessation treatment affects the smoking cessation efficacy, and evaluate predictors of adherence to smoking cessation treatment among patients with COPD in China.

\section{Method \\ Study Design}

This study was an exploratory analysis of data from an ongoing open-label randomized controlled trial (RCT) of varenicline and bupropion for smoking cessation among patients with COPD in China. This study was approved by Institutional Review Boards at China-Japan Friendship Hospital (No. 2018-108-K77) and registered in the Chinese Clinical Trial Registry (No. CTR1900021400, URL: http://www.chictr.org.cn).

This study was carried out at the China-Japan Friendship hospital in Beijing, China. Eligible participants were recruited via a trial site, a hotline of smoking cessation, advertisements in the community from February 2019 to June 2020.

All participants signed informed consent forms, received compensation for transportation, and all medication was distributed to participants free of charge.

\section{Participants}

Participants were included if they voluntarily participated in this trial and signed the informed consent form; they were diagnosed as $\mathrm{COPD}{ }^{1}$ they were diagnosed as tobacco dependence; ${ }^{16}$ they reported smoking for more than 5 years and smoking an average of more than 10 cigarettes per day during the previous year; exhaled carbon monoxide $(\mathrm{ECO}) \geq 10 \mathrm{ppm}$; they were required to be age $18-85$.

COPD was diagnosed as the post-bronchodilator ratio of forced expiratory volume in $1 \mathrm{~s}$ and forced vital capacity (FEV1/FVC) less than 0.70, according to the GOLD guideline. $^{1}$ 
Tobacco dependence was diagnosed if a minimum of three of the following six were met: 1) craving or a strong desire to use tobacco; 2) there is an unsuccessful effort to control the use of tobacco; 3) tobacco withdrawal after abrupt cessation or reduction of tobacco use; 4) tolerance, defined as the need for markedly increased amounts of tobacco to achieve the desired effect; 5) important social, or recreational activities or hobbies are given up or reduced because of tobacco use; and 6) tobacco use is continued despite recognizing the hazards of smoking. ${ }^{16}$

Participants were excluded if they had severe cardiovascular diseases (eg, acute myocardial infarction) or cerebrovascular diseases (eg, stroke); had neuropsychiatric disorders (eg, seizure and anorexia), had severe impairment of liver and kidney function (eg, renal failure), were pregnant or lactating women, had use of bupropion or varenicline within the last 30 days, or were allergic to them.

\section{Randomization and Blindness}

First, randomization was stratified by high/low nicotine metabolic rates; second, the allocation was assigned in a ratio of 1:1 in blocks of four patients (2/treatment/block) to ensure approximate balance. Third, a biostatistician, independent of the study used Proc Plan in SAS version 9.4 (SAS Institute) to generate a table of random digit to randomly assign the numbers to the two groups. (the number of the random seed is $87,654,321$ ).

To ensure random concealment, the group information assigned to each participant was put in a sealed, and opaque envelope. At the same time, the people who generated and saved the random allocation plan and the researchers who determined the selected participant were ensured to be different people.

Because of the different medication packaging, only statisticians were blinded to medication allocation.

\section{Intervention}

If eligible and willing to enroll, participants received 12 weeks of medication, and they were required to set a target quit date within 2 weeks after medication. Participants were required to make eight outpatient visits to ChinaJapan Friendship Hospital through 24 weeks. Face-toface visits and assessments were conducted at weeks 0 , $1,2,4,6,9,12$, and 24 after initiation of treatment. Participants received a counseling session for more than 60 minutes when they began medication at week 0 , and they also received up to $10 \mathrm{~min}$ of counseling at weeks 1 , $2,4,6,9,12$, and 24 .

The participants received the varenicline (purchased from Pfizer, Illertissen, Germany) $0.5 \mathrm{mg}$ once per day for the first 3 days; $0.5 \mathrm{mg}$ twice per day for the next 4 days; $1 \mathrm{mg}$ twice per day from day 8 .

The participants received the bupropion (purchased from Venturepharm, Hainan, China) one $150 \mathrm{mg}$ tablet per day.

This study was conducted according to the China Clinical Guideline for Tobacco Cessation. ${ }^{17}$

\section{Measures \\ Baseline Characteristics}

A questionnaire completed by participants at baseline provided measures of participant characteristics, including:

Demographics gender, age, nationality, education, marital status, etc.

Tobacco-related characteristics the number of cigarettes per day; duration of smoking; the Fagerstrom Test for Nicotine Dependence (FTND), which included six items, and the total score of $0-3,4-6$, and 7-10 meant mild, moderate, and severe tobacco dependence, respectively; ${ }^{18}$ previous quitting attempts; the Visual Analogue Scale was used to assess the self-efficacy and preparation in quitting smoking, and the score of 1-3, 4-6, 7-10 meant the level weak, medium, strong, respectively; ${ }^{19}$ whether recognizing the hazards of smoking, etc.

COPD-related features the duration of COPD; the GOLD stage I-IV, which were the classification of airflow limitation severity. FEV1 $\geq 80 \%$ predicted value (GOLD stage I), $50 \%$ predicted value $\leq \mathrm{FEV} 1<80 \%$ predicted value (stage II), $30 \%$ predicted value $\leq \mathrm{FEV} 1<50 \%$ (stage III), FEV $1<30 \%$ predicted value (stage IV) means mild, moderate, severe and very severe airflow limitation, respectively; ${ }^{1}$ St. George's Respiratory Questionnaire (SGRQ), which consisted of 50 questions divided into three subscales: symptom ( 8 questions), activity (16 questions), and impact (26 questions). All the questions had an attributed weight, and the total score was obtained from the sum of the three categories. ${ }^{20}$

\section{Adherence to Smoking Cessation Treatment}

Good adherence to smoking cessation treatment was defined as taking $>80 \%$ of medication across 12 weeks and face-to-face visits more than 5; otherwise, it was considered to have poor adherence. Pill count data were collected using a timeline follow-back method (TLFB) ${ }^{21}$ 
through self-report and by collecting used pill blisterpackages to confirm the accuracy of self-reports.

\section{Smoking Abstinence}

ECO was used to determine abstinence among participants who received smoking cessation treatment, with 10ppm used as a cut-point for abstinence. ${ }^{17} \mathrm{ECO}$ was collected inperson at weeks $0,1,2,4,6,9,12$, and 24 .

\section{FTND Score}

FTND score was also collected at weeks 1, 2, 4, 6, 9, 12, and 24.

\section{Statistical Analysis}

SPSS 26.0 software was used for all data statistics. Categorical measures were indicated with frequency and percentage, and continuous measures were expressed with Means \pm Standard Deviation $(\mathrm{X} \pm \mathrm{SD})$. For comparison of baseline characteristics between the two groups, the $t$-test was used for comparison which met Gaussian distribution and homogeneity of variance, and Wilcoxon Rank Sum Test was used for comparisons that did not meet homogeneity of variance. The chi-square test was used for categorical comparison.

Intention-to-treat (ITT) analysis was used, and the participants who were lost were recognized to be smokers. Logistic regression analysis was used to analyze the predictors of smoking cessation efficacy and adherence. The results were indicated as the Odds Ratio (OR) value and 95\% Confidence Interval (CI). P $<0.05$ was statistically significant.

\section{Results}

A total of 149 participants were assessed to be eligible, of which 13 did not meet the inclusion and exclusion criteria, and were excluded, and 136 participants were eventually recruited and analyzed. Eight and seven participants were lost in the varenicline and bupropion group, respectively (Figure 1).

Of these, 97.1\% (132/136) were male, 2.9\% (4/136) were female, and the total mean age (SD) was 62.16 (7.43) years; FTND score was 4.65 (2.45); cigarettes per day was 19.21 (10.67); duration of smoking was 42.00 (8.61) years; the percentage of COPD by GOLD stages I, II, III and IV was $75.7 \%(103 / 136), 19.1 \%(26 / 136), 5.1 \%(7 / 136)$ and $0 \%(0 / 136)$, respectively. There was no significant difference in baseline characteristics between the two groups ( $\mathrm{P}$ $>0.05$ ) (Table 1).
The abstinence rate at week 12 was significantly higher in the varenicline group (57.4\%) than in the bupropion group (35.3\%) $(\mathrm{OR}=2.62,95 \% \mathrm{CI} 1.25-5.52, \mathrm{P}<0.05$, the OR was adjusted for gender, age, nationality, education, and marital status). The comparison of abstinence rates at other time points is shown in Figure 2.

After adjusting for age and gender (Model 1), the logistic regression analysis of smoking cessation efficacy showed that the FTND score, the number of cigarettes per day, adherence to smoking cessation treatment, and smoking cessation medication were correlated with the efficacy of smoking cessation $(\mathrm{P}<0.05)$. The same results were obtained after adjusting for age, gender, nationality, education, and marital status (Model 2) (Table S1, as shown in the Appendix).

Because good adherence to smoking cessation treatment significantly improved the efficacy of smoking cessation $(\mathrm{OR}=9.60,95 \% \mathrm{CI}$ 4.02-22.96, $\mathrm{P}<0.001)$, we further compared the differences in treatment adherence between the two groups and the predictors of adherence. The good adherence group (66/136) and poor adherence group (70/136) accounted for $48.5 \%$ and $51.5 \%$ of the total participants, respectively. In both groups, good adherence decreased as treatment time extended (Figure 3A), and rates of good adherence were similar in the varenicline and bupropion groups (Figure 3B). Overall, $71 \%$ of participants with good adherence had quit smoking vs $23 \%$ of those with poor adherence (Figure 3C). At each visit, the FTND score of participants with good adherence was lower than that with poor adherence (Figure 3D).

After adjusting for age and gender (Model 1), the logistic regression analysis of adherence showed that older age, higher education level, having more attempts to quit, stronger self-efficacy and preparation, recognizing the hazards of smoking, longer duration of COPD, and higher SGRQ symptom, impact, and total score were relevant to good adherence $(\mathrm{P}<0.05)$. Besides higher SGRQ activity scores related to good adherence, the same results were obtained after adjusting for age, gender, nationality, education, and marital status (Model 2) (Table 2).

\section{Discussion}

To the best of our knowledge, this was the first study to assess adherence to smoking cessation treatment among patients with COPD in China, and our study found that good adherence to smoking cessation treatment will increase smoking cessation efficacy and predictors of adherence were evaluated. 


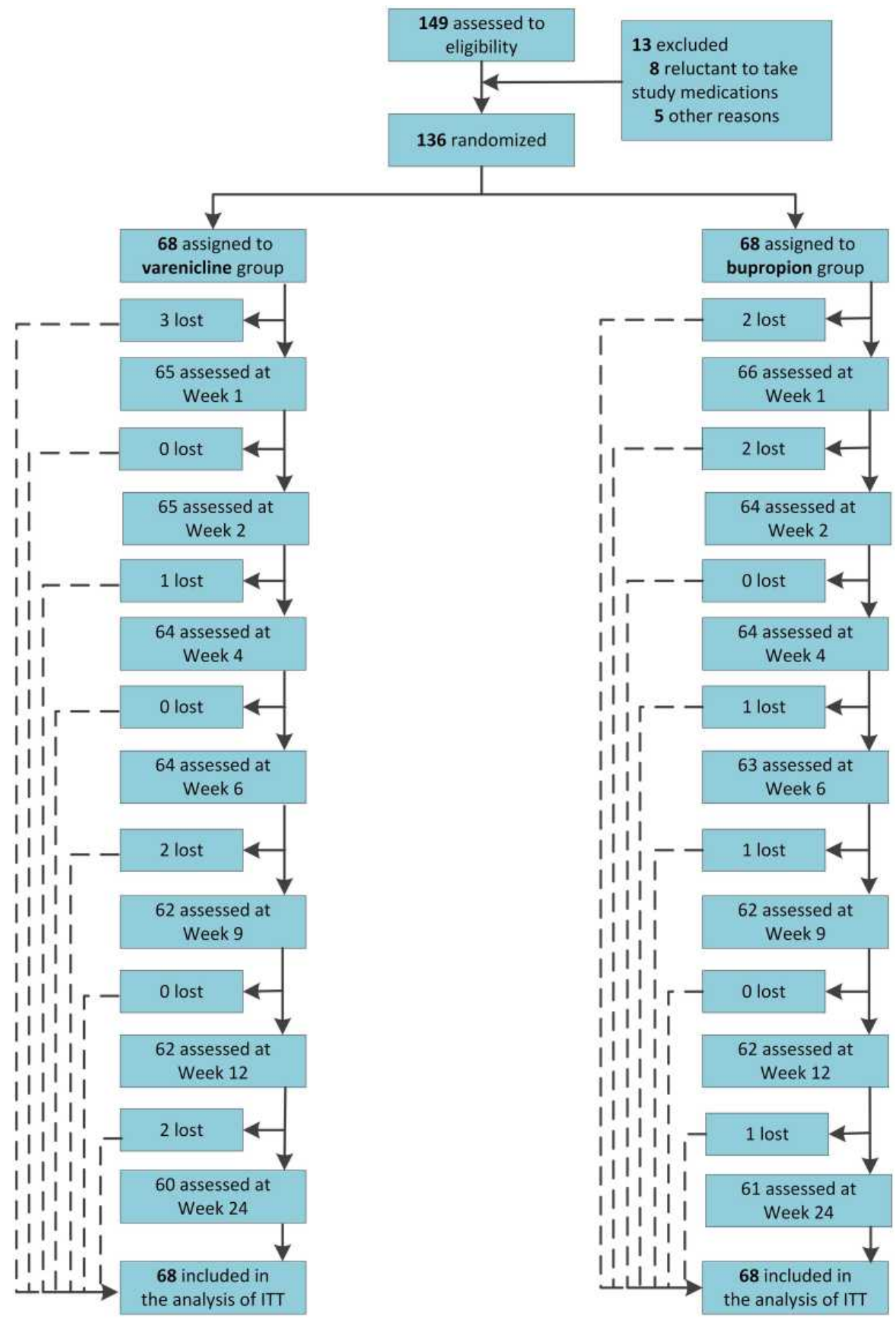

Figure I Flowchart of participants.

Abbreviation: ITT, intention to treatment.

Adherence has been assessed by many measures, Starts with Knowledge questionnaire. ${ }^{12}$ We applied but none can be considered as a gold standard. Different measures have been used to describe the adherence, ranging from the proportion of medication consumed, dichotomized to evaluate a cutoff (ie, $80 \%$ of pills used) to questionnaires such as the Adherence measures with an $80 \%$ cutoff, which is widely used to varenicline, bupropion and oral medication for chronic diseases. ${ }^{3,22,23}$

To date, the proportion of good adherence to smoking cessation treatment varied in different studies. $^{24}$ For 
Table I Characteristics of Participants

\begin{tabular}{|c|c|c|c|c|}
\hline Characteristics & Varenicline $(n=68)$ & Bupropion $(n=68)$ & Total & $P$ value \\
\hline Gender & & & & 0.31 \\
\hline Male & 67 (98.5\%) & $65(95.6 \%)$ & I 32 (97.1\%) & \\
\hline Female & I (I.5\%) & $3(4.4 \%)$ & $4(2.9 \%)$ & \\
\hline Age, y & & & & 0.69 \\
\hline$\leq 55$ & $10(14.7 \%)$ & $\mathrm{II}(16.2 \%)$ & $21(15.4 \%)$ & \\
\hline $56-60$ & 14 (20.6\%) & II (I6.2\%) & $25(18.4 \%)$ & \\
\hline $6 I-65$ & $24(35.3 \%)$ & 19 (27.9\%) & 43 (31.6\%) & \\
\hline $66-70$ & $16(23.5 \%)$ & $20(29.4 \%)$ & $36(26.5 \%)$ & \\
\hline$>70$ & $4(5.9 \%)$ & $7(10.3 \%)$ & II (8.1\%) & \\
\hline Nationality & & & & 0.17 \\
\hline Han & $67(98.5 \%)$ & $64(94.1 \%)$ & $|3|(96.3 \%)$ & \\
\hline Non-Han & $\mathrm{I}(\mathrm{I} .5 \%)$ & $4(5.9 \%)$ & $5(3.7 \%)$ & \\
\hline Education & & & & 0.67 \\
\hline Primary school and below & 8 (II.8\%) & $5(7.4 \%)$ & $13(9.6 \%)$ & \\
\hline Middle school & $40(58.8 \%)$ & $43(63.2 \%)$ & $83(61.0 \%)$ & \\
\hline College and above & $20(29.4 \%)$ & $20(29.4 \%)$ & 40 (29.4\%) & \\
\hline Marital status & & & & 0.30 \\
\hline Married & 67 (98.5\%) & $64(94.1 \%)$ & |3| (96.3\%) & \\
\hline Divorced, widowed, and separated & $\mathrm{I}(\mathrm{I} .5 \%)$ & $2(2.9 \%)$ & $3(2.2 \%)$ & \\
\hline Unmarried & $0(0.0 \%)$ & $2(2.9 \%)$ & $2(1.5 \%)$ & \\
\hline Cigarettes per day & & & & 0.25 \\
\hline$\leq 10$ & $14(20.6 \%)$ & $20(29.4 \%)$ & $34(25.0 \%)$ & \\
\hline $\mathrm{II}-20$ & $38(55.9 \%)$ & $40(58.8 \%)$ & 78 (57.4\%) & \\
\hline $21-30$ & $8(11.8 \%)$ & $3(4.4 \%)$ & II (8.1\%) & \\
\hline$>30$ & $8(11.8 \%)$ & $5(7.4 \%)$ & $13(9.6 \%)$ & \\
\hline Duration of smoking, $y$ & & & & 0.17 \\
\hline$\leq 30$ & $5(7.4 \%)$ & $10(14.7 \%)$ & $15(11.0 \%)$ & \\
\hline $3 I-40$ & $24(35.3 \%)$ & $22(32.4 \%)$ & 46 (33.8\%) & \\
\hline $4 I-50$ & $36(52.9 \%)$ & $28(4 I .2 \%)$ & $64(47.1 \%)$ & \\
\hline$>50$ & $3(4.4 \%)$ & $8(11.8 \%)$ & II (8.1\%) & \\
\hline FTND score & & & & 0.29 \\
\hline $0-3$ & $21(30.9 \%)$ & $29(42.6 \%)$ & $50(36.8 \%)$ & \\
\hline $4-6$ & $23(33.8 \%)$ & $22(32.4 \%)$ & $45(33.1 \%)$ & \\
\hline $7-10$ & $24(35.3 \%)$ & $17(25.0 \%)$ & $4 I$ (30.1\%) & \\
\hline Previous quitting attempts & & & & 0.90 \\
\hline None & $23(33.8 \%)$ & $22(32.4 \%)$ & $45(33.1 \%)$ & \\
\hline 1 & 15 (22.1\%) & $18(26.5 \%)$ & $33(24.3 \%)$ & \\
\hline $2-3$ & $23(33.8 \%)$ & $20(29.4 \%)$ & $43(31.6 \%)$ & \\
\hline$\geq 4$ & $7(10.3 \%)$ & $8(11.8 \%)$ & $15(11.0 \%)$ & \\
\hline Self-efficacy in quitting smoking & & & & 0.17 \\
\hline Weak & $15(22.1 \%)$ & II (16.2\%) & $26(19.1 \%)$ & \\
\hline Medium & $23(33.8 \%)$ & $16(23.5 \%)$ & 39 (28.7\%) & \\
\hline Strong & $30(44.1 \%)$ & $41(60.3 \%)$ & 71 (52.2\%) & \\
\hline Preparation in quitting smoking & & & & 0.32 \\
\hline Weak & $14(20.6 \%)$ & $17(25.0 \%)$ & 31 (22.8\%) & \\
\hline Medium & $17(25.0 \%)$ & $10(14.7 \%)$ & 27 (19.9\%) & \\
\hline
\end{tabular}

(Continued) 
Table I (Continued).

\begin{tabular}{|c|c|c|c|c|}
\hline Characteristics & Varenicline $(n=68)$ & Bupropion $(n=68)$ & Total & $P$ value \\
\hline Strong & 37 (54.4\%) & $41(60.3 \%)$ & 78 (57.4\%) & \\
\hline $\begin{array}{l}\text { Whether recognizing hazards of smoking } \\
\text { Yes } \\
\text { No }\end{array}$ & $\begin{array}{l}13(19.1 \%) \\
55(80.9 \%)\end{array}$ & $\begin{array}{l}\text { I7 (25.0\%) } \\
5 I(75.0 \%)\end{array}$ & $\begin{array}{c}30(22.1 \%) \\
106(77.9 \%)\end{array}$ & 0.41 \\
\hline $\begin{array}{l}\text { Duration of COPD, y } \\
\qquad 1 \\
1-4 \\
>4\end{array}$ & $\begin{array}{l}55(80.9 \%) \\
4(5.9 \%) \\
9(13.2 \%)\end{array}$ & $\begin{array}{c}50(73.5 \%) \\
12(17.6 \%) \\
6(8.8 \%)\end{array}$ & $\begin{array}{l}105(77.2 \%) \\
16(11.8 \%) \\
15(11.0 \%)\end{array}$ & 0.09 \\
\hline $\begin{array}{l}\text { GOLD stage } \\
\text { Stage I } \\
\text { Stage II } \\
\text { Stage III } \\
\text { Stage IV }\end{array}$ & $\begin{array}{c}50(73.5 \%) \\
14(20.6 \%) \\
4(5.9 \%) \\
0(0 \%)\end{array}$ & $\begin{array}{c}53(77.9 \%) \\
12(17.6 \%) \\
3(4.4 \%) \\
0(0 \%)\end{array}$ & $\begin{array}{c}103(75.7 \%) \\
26(19.1 \%) \\
7(5.1 \%) \\
0(0 \%)\end{array}$ & 0.68 \\
\hline $\begin{array}{l}\text { SGRQ } \\
\text { Symptom score } \\
\text { Activity score } \\
\text { Impact score } \\
\text { Total score }\end{array}$ & $\begin{array}{l}28.95(23.02) \\
12.20(14.57) \\
12.26(13.68) \\
15.37(14.11)\end{array}$ & $\begin{array}{l}24.49(18.34) \\
\text { I I.54 (I7.62) } \\
10.49(14.95) \\
\text { I3.46 (I4.23) }\end{array}$ & $\begin{array}{l}26.71 \text { (20.83) } \\
\text { II.87 (I6.II) } \\
\text { II.37 (I4.30) } \\
\text { I4.42 (I4.15) }\end{array}$ & $\begin{array}{l}0.22 \\
0.81 \\
0.48 \\
0.41\end{array}$ \\
\hline
\end{tabular}

Abbreviations: FTND, Fagerstrom Test for Nicotine Dependence; COPD, chronic obstructive pulmonary disease; GOLD, Global Initiative for Chronic Obstructive Lung Disease; SGRQ, St. George's Respiratory Questionnaire.

example, an RCT among the American adolescent population found that $74.24 \%$ of participants had good adherence to bupropion. ${ }^{25}$ Nevertheless, in an observational study of the Dutch population, good adherence was found in only $14.3 \%$ of the participants using varenicline. ${ }^{26}$ Our study found that only about half of the COPD patients in China had good adherence to smoking cessation treatment, which

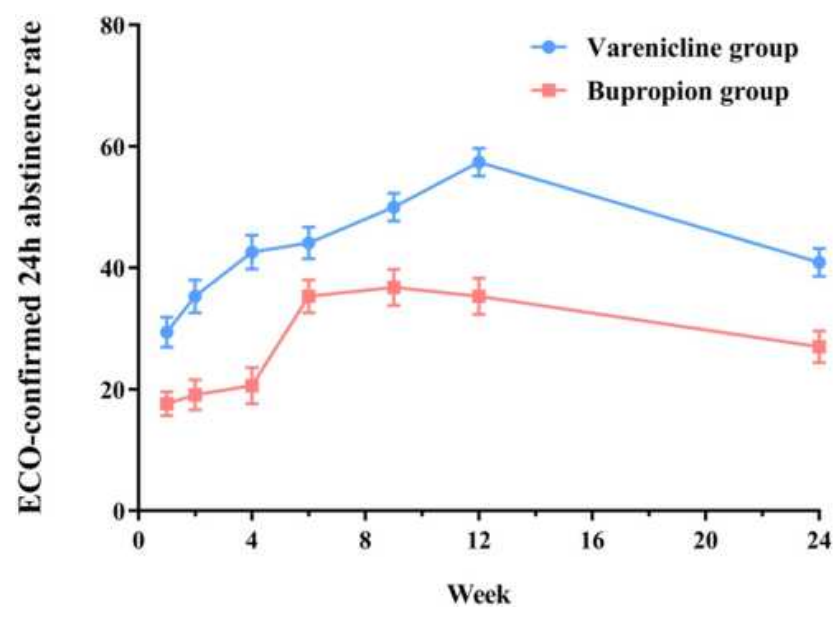

Figure 2 Comparison of abstinence rate between varenicline and bupropion at different time points.

Abbreviation: ECO, exhaled carbon monoxide. was similar to the results of RCTs among AIDS patients $(56 \%)^{3}$ and cancer patients $(56 \%)^{27}$ in the United States. The variation is likely due to the differences in the definition of adherence, interventions, adjunctive supports, and population selection.

Our study founded that the abstinence rate of varenicline at the end of treatment was significantly higher than bupropion, which was consistent with the results of previous studies. ${ }^{6,28}$ Importantly, good adherence to smoking cessation treatment among patients with COPD in China significantly improved the smoking cessation efficacy, which was consistent with studies in the other population. $^{15,29}$

At the same time, our study identified the predictors of adherence. The preventable factors include self-efficacy and preparation in quitting smoking and cognition of smoking hazards, and the non-preventable factors included age, education, and previous quitting attempts, which was consistent with the results of studies in the general population. ${ }^{12}$ For example, older age is a known predictor of good adherence to smoking cessation treatment, which was consistent with the results of previous studies. ${ }^{30,31}$ Older people especially those older than 70, usually had good adherence to smoking cessation treatment. This might be due to older people having 

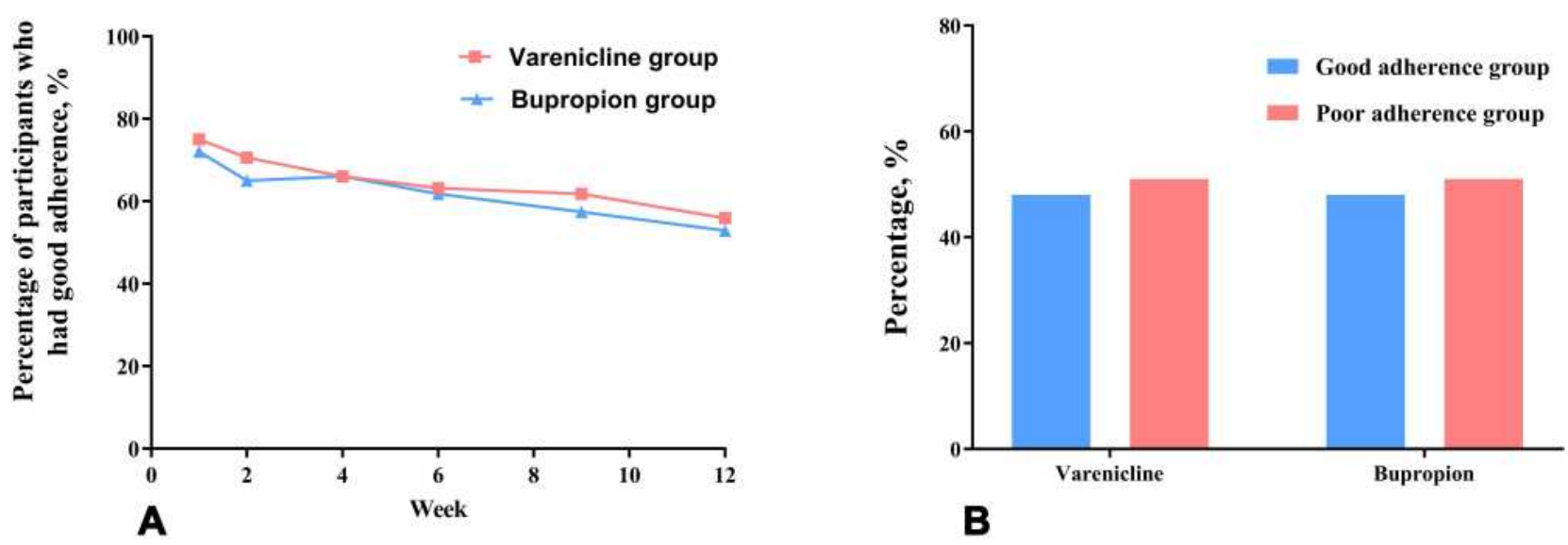

\section{B}
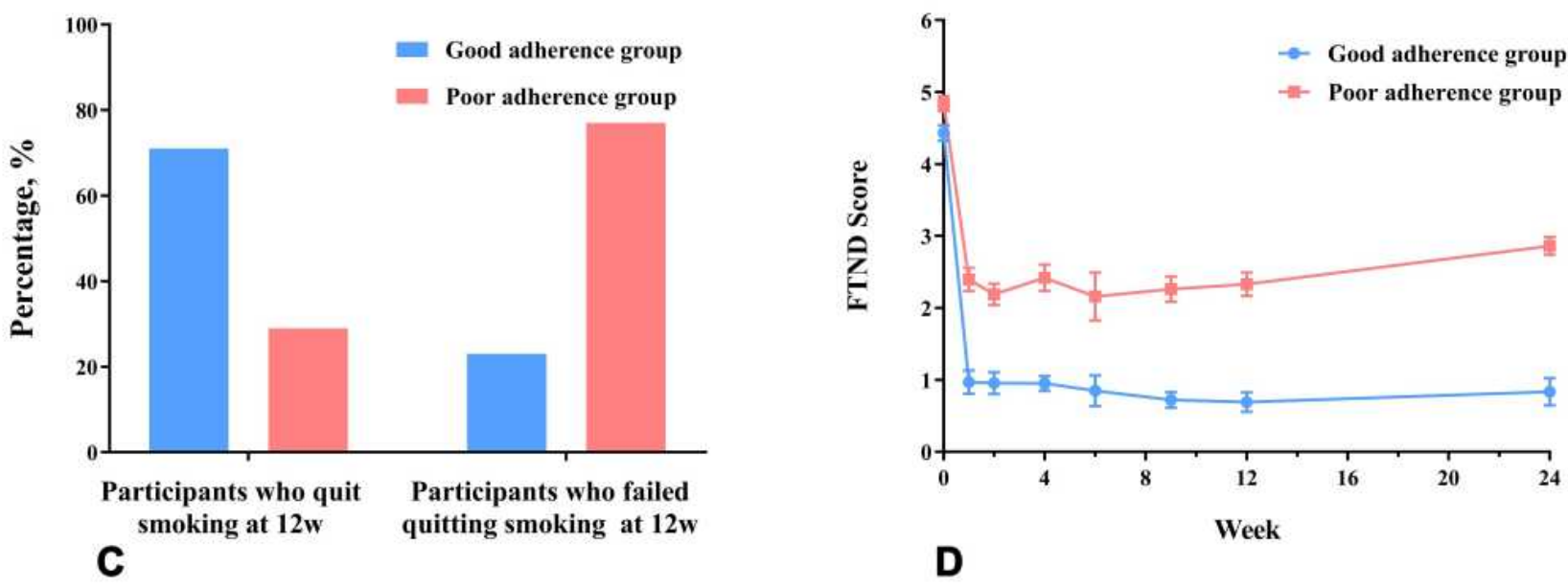

D

Figure 3 Adherence to smoking cessation treatment and its relationship with medication use and the efficacy of smoking cessation.

Notes: $(\mathbf{A})$ The overall treatment adherence; $(\mathbf{B})$ the relationship between adherence to smoking cessation treatment and medication use; (C) the relationship between adherence and smoking cessation efficacy; (D) FTND Score change.

Abbreviation: FTND, Fagerstrom Test for Nicotine Dependence.

more chronic diseases, more experience with medications, deeper recognition of smoking hazards and more previous quitting attempts.

Importantly, we found that a longer duration of COPD and higher SGRQ score were relevant to good adherence to smoking cessation treatment. Previous studies $^{32-34}$ found the relationship between COPDrelated features and the efficacy of smoking cessation. For example, the study by Tøttenborg et al found that the higher GOLD stage, the better the efficacy of smoking cessation. ${ }^{33}$ With the extension of the duration of COPD, respiratory symptoms were aggravated, activities were gradually restricted, and the recognition of the smoking hazards was deeper. Therefore, deterioration of health could increase the adherence to smoking cessation treatment to improve the efficacy of smoking cessation.
However, our study did not find a correlation between adherence to smoking cessation treatment and tobacco dependence, which was consistent with the result of the study by Okuyemi et al. ${ }^{35}$ Studies by Vaz et $\mathrm{al}^{36}$ and Balmford et $\mathrm{al}^{37}$ found that a positive correlation between adherence to smoking cessation treatment and tobacco dependence, while the study by Hood et $\mathrm{al}^{38}$ found a negative correlation. The inconsistency of results might be due to the differences in the interventions and population selection.

This study has important clinical implications. Considering that good adherence significantly increased the efficacy of smoking cessation, we call on the medical community to pay attention to the adherence to smoking cessation treatment among patients with COPD. Healthcare providers play a very important role in helping patients to quit smoking, ${ }^{39}$ and they should provide 
Table 2 Logistic Regression Analyses of Participants' Characteristics to Predict Adherence

\begin{tabular}{|c|c|c|c|c|}
\hline \multirow{2}{*}{ Variables } & \multicolumn{2}{|c|}{ Model I } & \multicolumn{2}{|c|}{ Model 2} \\
\hline & $(\mathrm{OR}, 95 \% \mathrm{CI})$ & $P$ value & $(\mathrm{OR}, 95 \% \mathrm{CI})$ & $P$ value \\
\hline \multicolumn{5}{|l|}{ Gender } \\
\hline Male & Ref & & Ref & \\
\hline Female & $1.28(0.16-10.25)$ & 0.815 & $1.80(0.15-21.40)$ & 0.643 \\
\hline \multicolumn{5}{|l|}{ Age, y } \\
\hline$<56$ & Ref & & Ref & \\
\hline $56-60$ & $1.96(0.57-6.75)$ & 0.284 & $1.65(0.45-5.98)$ & 0.448 \\
\hline $6 I-65$ & $2.39(0.78-7.36)$ & 0.129 & I.8I (0.56-5.89) & 0.325 \\
\hline $66-70$ & $2.80(0.88-8.88)$ & 0.081 & $2.56(0.76-8.67)$ & 0.130 \\
\hline$>70$ & II.24 (I.85-68.24) & 0.009 & $9.93(1.47-67.29)$ & 0.019 \\
\hline \multicolumn{5}{|l|}{ Nationality } \\
\hline Han & Ref & & Ref & \\
\hline Non-Han & $0.00(0.00-0.00)$ & 0.999 & $0.00(0.00-0.00)$ & 0.999 \\
\hline \multicolumn{5}{|l|}{ Education } \\
\hline Primary school and below & Ref & & Ref & \\
\hline Middle school & $2.14(0.52-8.8 I)$ & 0.294 & $1.98(0.48-8.24)$ & 0.347 \\
\hline College and above & $5.52(1.22-24.98)$ & 0.027 & $5.31(1.17-24.20)$ & 0.031 \\
\hline \multicolumn{5}{|l|}{ Marital status } \\
\hline Married & Ref & & Ref & \\
\hline Divorced, widowed, separated & $1.64(0.09-29.73)$ & 0.740 & $2.17(0.12-38.56)$ & 0.597 \\
\hline Unmarried & $0.46(0.04-5.31)$ & 0.531 & $0.64(0.05-7.61)$ & 0.722 \\
\hline \multicolumn{5}{|l|}{ Cigarettes per day } \\
\hline$\leq 10$ & Ref & & Ref & \\
\hline $\mathrm{II}-20$ & I.0I (0.43-2.38) & 0.982 & I.0I (0.4I-2.50) & 0.989 \\
\hline $21-30$ & $1.34(0.32-5.56)$ & 0.692 & $1.95(0.40-9.57)$ & 0.410 \\
\hline$>30$ & $0.56(0.14-2.27)$ & 0.413 & $0.75(0.17-3.25)$ & 0.697 \\
\hline \multicolumn{5}{|l|}{ Duration of smoking, $y$} \\
\hline$\leq 30$ & Ref & & Ref & \\
\hline $31-40$ & $1.62(0.42-6.37)$ & 0.486 & $1.66(0.40-6.84)$ & 0.486 \\
\hline $4 I-50$ & $1.33(0.34-5.23)$ & 0.688 & $1.39(0.33-5.90)$ & 0.656 \\
\hline$>50$ & $0.73(0.10-5.18)$ & 0.753 & I.I5(0.14-9.53) & 0.896 \\
\hline \multicolumn{5}{|l|}{ FTND score } \\
\hline $0-3$ & Ref & & Ref & \\
\hline $4-6$ & $1.26(0.54-2.94)$ & 0.591 & $1.40(0.57-3.43)$ & 0.467 \\
\hline $7-10$ & $0.53(0.22-1.30)$ & 0.166 & $0.60(0.23-1.56)$ & 0.294 \\
\hline \multicolumn{5}{|l|}{ Previous quitting attempts } \\
\hline None & Ref & & Ref & \\
\hline I & $0.69(0.26-1.8 I)$ & 0.448 & $0.77(0.28-2.14)$ & 0.621 \\
\hline $2-3$ & $0.82(0.34-1.97)$ & 0.650 & $0.65(0.25-1.69)$ & 0.374 \\
\hline$\geq 4$ & $5.46(1.28-23.30)$ & 0.022 & $5.17(1.05-25.50)$ & 0.044 \\
\hline \multicolumn{5}{|l|}{ Self-efficacy in quitting smoking } \\
\hline Weak & Ref & & Ref & \\
\hline Medium & $1.45(0.48-4.38)$ & 0.506 & $\mathrm{I} .45(0.47-4.5 \mathrm{I})$ & 0.519 \\
\hline Strong & $3.6 \mathrm{I}(1.34-9.72)$ & 0.011 & $2.85(1.02-7.95)$ & 0.046 \\
\hline
\end{tabular}

(Continued) 
Table 2 (Continued).

\begin{tabular}{|c|c|c|c|c|}
\hline \multirow[t]{2}{*}{ Variables } & \multicolumn{2}{|c|}{ Model I } & \multicolumn{2}{|c|}{ Model 2} \\
\hline & $(\mathrm{OR}, 95 \% \mathrm{CI})$ & $P$ value & (OR, 95\% Cl) & $P$ value \\
\hline \multicolumn{5}{|c|}{ Preparation to quitting smoking } \\
\hline Weak & Ref & & Ref & \\
\hline Medium & $1.92(0.60-6.12)$ & 0.268 & $2.37(0.70-8.00)$ & 0.164 \\
\hline Strong & $3.93(1.52-10.17)$ & 0.005 & $4.03(1.45-11.19)$ & 0.008 \\
\hline \multicolumn{5}{|c|}{ Whether recognizing hazards of smoking } \\
\hline Yes & Ref & & Ref & \\
\hline No & $0.39(0.16-0.96)$ & 0.040 & $0.37(0.14-0.95)$ & 0.039 \\
\hline \multicolumn{5}{|c|}{ Duration of COPD, y } \\
\hline$<1$ & Ref & & Ref & \\
\hline $1-4$ & $1.53(0.47-5.03)$ & 0.483 & $1.61(0.44-5.95)$ & 0.475 \\
\hline$>4$ & $5.26(1.37-20.17)$ & 0.015 & $7.09(1.77-28.37)$ & 0.006 \\
\hline \multicolumn{5}{|l|}{ SGRQ } \\
\hline Symptom score & $1.02(1.00-1.04)$ & 0.022 & $1.03(1.01-1.05)$ & 0.012 \\
\hline Activity score & $1.02(1.00-1.05)$ & 0.074 & $1.03(1.00-1.05)$ & 0.038 \\
\hline Impact score & $1.04(1.01-1.08)$ & 0.008 & $1.05(1.02-1.08)$ & 0.004 \\
\hline Total score & $1.04(1.01-1.07)$ & 0.008 & $1.05(1.02-1.08)$ & 0.003 \\
\hline \multicolumn{5}{|l|}{ Medication } \\
\hline Bupropion & Ref & & Ref & \\
\hline Varenicline & $1.07(0.53-2.17)$ & 0.844 & $1.27(0.60-2.71)$ & 0.537 \\
\hline
\end{tabular}

Notes: Model I was adjusted for gender and age; Model 2 was adjusted for gender, age, nationality, education, and marital status.

Abbreviations: FTND, Fagerstrom Test for Nicotine Dependence; COPD, chronic obstructive pulmonary disease; SGRQ, St. George's Respiratory Questionnaire.

adequate education to patients on the importance of adherence to smoking cessation treatment. What's more, health professionals can improve adherence to smoking cessation treatment among patients with COPD via preventable characteristics such as improving self-efficacy in quitting smoking and deepening cognition of smoking hazards. It appears that this will be particularly important for individuals with characteristics (eg, younger age and lower education level), which were associated with poor adherence.

This study has several strengths, including being the first study to assess adherence to smoking cessation among patients with COPD in China, stringent procedure design, assessment of medication use at multiple time points, ECO-confirmed abstinence rates, as well as assessments of multiple variables that could affect adherence to smoking cessation. Nevertheless, our study also has limitations. First, compared with biological measures such as blood levels of medication metabolites, self-report may not be ideal. However, most studies utilized self-report as the primary measure of adherence because self-report is convenient and easy to administer. ${ }^{11}$ Second, most participants in our study were patients with moderate to mild COPD, so the results may not be directly extrapolated to patients with more severe COPD. Third, varenicline and bupropion provided free to participants, and the results could have been different if participants paid for them because cost-prohibitive prices were identified as barriers to adherence. ${ }^{40}$ Last, although we have considered many predictors, other potential genetic factors such as genephenotype of cytochrome P450 $2 \mathrm{~A}^{41}$ were not assessed.

\section{Conclusion}

To our best knowledge, this is the first study to evaluate adherence to smoking cessation among patients with COPD in China. Our study found that only about half of COPD smokers had good adherence to smoking cessation treatment. Good adherence to smoking cessation treatment significantly improved the smoking cessation efficacy, and predictors of adherence were evaluated. We call on the medical community to pay attention to the adherence to smoking cessation treatment among patients with COPD. 


\section{Abbreviations}

COPD, chronic obstructive pulmonary disease; GOLD, Global Initiative for Chronic Obstructive Lung Disease; RCT, randomized controlled trial; FEV1, forced expiratory volume in 1s; FVC, forced vital capacity; SGRQ, St. George's Respiratory Questionnaire; ECO, Exhaled carbon monoxide; FTND, Fagerstrom Test for Nicotine Dependence; OR, odds ratio; CI, confidence interval.

\section{Data Sharing Statement}

The data analyzed in the current study are not publicly available but may be available from the corresponding author Dr Xiao upon reasonable request.

\section{Acknowledgment}

We thank all the patients for their participation in this study.

\section{Author Contributions}

All authors made substantial contributions to conception and design, acquisition of data, or analysis and interpretation of data; took part in drafting the article or revising it critically for important intellectual content; agreed to submit to the current journal; gave final approval of the version to be published; and agree to be accountable for all aspects of the work.

\section{Funding}

This study was supported by the Capital Health Development Research Project in China (Grant No. 2018-2-4066), the National Natural Science Foundation of China (Grant No. 81720108001) and the National Key R\&D Program of China (Grant No. 2017YFC1309400).

\section{Disclosure}

The authors report no conflicts of interest in this work.

\section{References}

1. The GOLD Science Commitee. Global strategy for the diagnosis, management, and prevention of chronic pulmonary disease (2021 REPORT); 2020. Available from: https:/goldcopd.org/wp-content/ uploads/2020/11/GOLD-REPORT-2021-v1.0-16Nov20_WMV.pdf. Accessed November 18, 2020.

2. World Health Organization. The top 10 causes of death; 2018. Available from: http://www.who.int/en/newsroom/fact-sheets/detail/ the-top-10-causes-of-death. Accessed November 28, 2020.

3. Shelley D, Tseng TY, Gonzalez M, et al. Correlates of adherence to varenicline among HIV+ smokers. Nicotine Tob Res. 2015;17(8):968974. doi:10.1093/ntr/ntv068

4. Wang $\mathrm{C}, \mathrm{Xu} \mathrm{J}$, Yang $\mathrm{L}$, et al. Prevalence and risk factors of chronic obstructive pulmonary disease in China (the China Pulmonary Health [CPH] study): a national cross-sectional study. Lancet. 2018;391 (10131):1706-1717. doi:10.1016/S0140-6736(18)30841-9
5. Stead LF, Koilpillai P, Fanshawe TR, et al. Combined pharmacotherapy and behavioural interventions for smoking cessation. Cochrane Database Syst Rev. 2016;3:CD008286. doi:10.1002/14651858. CD008286.pub3

6. Cahill K, Stevens S, Perera R, et al. Pharmacological interventions for smoking cessation: an overview and network meta-analysis. Cochrane Database Syst Rev. 2013;5:CD009329. doi:10.1002/ 14651858.CD009329.pub2

7. van Eerd EA, van der Meer RM, van Schayck OC, et al. Smoking cessation for people with chronic obstructive pulmonary disease. Cochrane Database Syst Rev. 2016;8:CD010744. doi:10.1002/ 14651858.CD010744.pub2

8. Vrijens B, De Geest S, Hughes DA, et al. A new taxonomy for describing and defining adherence to medications. $\mathrm{Br} J$ Clin Pharmacol. 2012;73(5):691-705. doi:10.1111/j.13652125.2012.04167.x

9. Solberg LI, Parker ED, Foldes SS, et al. Disparities in tobacco cessation medication orders and fills among special populations. Nicotine Tob Res. 2010;12(2):144-151. doi:10.1093/ntr/ntp187

10. Zeng F, Chen CI, Mastey V, et al. Effects of copayment on initiation of smoking cessation pharmacotherapy: an analysis of varenicline reversed claims. Clin Ther. 2011;33(2):225-234. doi:10.1016/j. clinthera.2011.02.013

11. Pacek LR, McClernon FJ, Bosworth HB. Adherence to pharmacological smoking cessation interventions: a literature review and synthesis of correlates and barriers. Nicotine Tob Res. 2018;20(10):11631172. doi: $10.1093 / \mathrm{ntr} / \mathrm{ntx} 210$

12. van Eerd EA, van Rossem CR, Spigt MG, et al. Do we need tailored smoking cessation interventions for smokers with COPD? A comparative study of smokers with and without COPD regarding factors associated with tobacco smoking. Respiration. 2015;90(3):211-219. doi: $10.1159 / 000398816$

13. Borrelli B, Hayes RB, Dunsiger S, et al. Risk perception and smoking behavior in medically ill smokers: a prospective study. Addiction. 2010;105(6):1100-1108. doi:10.1111/j.13600443.2010.02900.x

14. Helleberg M, Afzal S, Kronborg G, et al. Mortality attributable to smoking among HIV-1-infected individuals: a nationwide, population-based cohort study. Clin Infect Dis. 2013;56(5):727-734. doi:10.1093/cid/cis933

15. Crawford G, Weisbrot J, Bastian J, et al. Predictors of varenicline adherence among cancer patients treated for tobacco dependence and its association with smoking cessation. Nicotine Tob Res. 2019;21 (8):1135-1139. doi:10.1093/ntr/nty133

16. World Health Organization. The ICD-10 classification of mental and behavioural disorders: clinical descriptions and diagnostic guidelines; 1992.

17. China National Health and Family Planning Commission. China clinical guidelines for tobacco cessation; 2015.

18. Heatherton TF, Kozlowski LT, Frecker RC, et al. The fagerstrom test for nicotine dependence: a revision of the fagerstrom tolerance questionnaire. $\mathrm{Br} J$ Addict. 1991;86(9):1119-1127. doi:10.1111/j.13600443.1991.tb01879.x

19. Sung YT, Wu JS. The visual analogue scale for rating, ranking and paired-comparison (VAS-RRP): a new technique for psychological measurement. Behav Res Methods. 2018;50(4):1694-1715. doi:10.3758/s13428-018-1041-8

20. Jones P. St. George's respiratory questionnaire manual. 2009.

21. Hjorthoj CR, Hjorthoj AR, Nordentoft M. Validity of timeline followback for self-reported use of cannabis and other illicit substancessystematic review and meta-analysis. Addict Behav. 2012;37(3):225233. doi:10.1016/j.addbeh.2011.11.025

22. DiMatteo MR, Giordani PJ, Lepper HS, et al. Patient adherence and medical treatment outcomes: a meta-analysis. Med Care. 2002;40 (9):794-811. doi:10.1097/00005650-200209000-00009 
23. Browning KK, Wewers ME, Ferketich AK, et al. Adherence to tobacco dependence treatment among HIV-infected smokers. AIDS Behav. 2016;20(3):608-621. doi:10.1007/s10461-015-1059-1

24. Moulding HD, Friedman DP, Curtis M, et al. Revisiting anaplastic astrocytomas I: an expansive growth pattern is associated with a better prognosis. J Magn Reson Imaging. 2008;28(6):1311-1321. doi:10.1002/jmri.21593

25. Leischow SJ, Muramoto ML, Matthews E, et al. Adolescent smoking cessation with bupropion: the role of adherence. Nicotine Tob Res. 2016;18(5):1202-1205. doi:10.1093/ntr/ntv179

26. van Boven JF, Vemer P. Higher adherence during reimbursement of pharmacological smoking cessation treatments. Nicotine Tob Res. 2016;18(1):56-63. doi:10.1093/ntr/ntv064

27. Carroll AJ, Veluz-Wilkins AK, Blazekovic S, et al. Cancer-related disease factors and smoking cessation treatment: analysis of an ongoing clinical trial. Psychooncology. 2018;27(2):471-476. doi: $10.1002 /$ pon.4483

28. Gonzales D, Jorenby DE, Brandon TH, et al. Immediate versus delayed quitting and rates of relapse among smokers treated successfully with varenicline, bupropion SR or placebo. Addiction. 2010;105 (11):2002-2013. doi:10.1111/j.1360-0443.2010.03058.x

29. Grandi SM, Eisenberg MJ, Joseph L, et al. Cessation treatment adherence and smoking abstinence in patients after acute myocardial infarction. Am Heart J. 2016;173:35-40. doi:10.1016/j. ahj.2015.12.003

30. Catz SL, Jack LM, McClure JB, et al. Adherence to varenicline in the COMPASS smoking cessation intervention trial. Nicotine Tob Res. 2011;13(5):361-368. doi:10.1093/ntr/ntr003

31. Hays JT, Leischow SJ, Lawrence D, et al. Adherence to treatment for tobacco dependence: association with smoking abstinence and predictors of adherence. Nicotine Tob Res. 2010;12(6):574-581. doi: $10.1093 / \mathrm{ntr} / \mathrm{ntq} 047$

32. Pezzuto A, Carico E. Effectiveness of smoking cessation in smokers with COPD and nocturnal oxygen desaturation: functional analysis. Clin Respir J. 2020;14(1):29-34. doi:10.1111/crj.13096
33. Tøttenborg SS, Thomsen RW, Johnsen SP, et al. Determinants of smoking cessation in patients with COPD treated in the outpatient setting. Chest. 2016;150(3):554-562. doi:10.1016/j. chest.2016.05.020

34. Kupiainen H, Kinnula VL, Lindqvist A, et al. Successful smoking cessation in COPD: association with comorbidities and mortality. Pulm Med. 2012;2012:725024. doi:10.1155/2012/725024

35. Okuyemi KS, Zheng $\mathrm{H}$, Guo $\mathrm{H}$, et al. Predictors of adherence to nicotine gum and counseling among African-American light smokers. J Gen Intern Med. 2010;25(9):969-976. doi:10.1007/s11606-0101386-X

36. Vaz LR, Aveyard P, Cooper S, et al. The association between treatment adherence to nicotine patches and smoking cessation in pregnancy: a secondary analysis of a randomized controlled trial. Nicotine Tob Res. 2016;18(10):1952-1959. doi:10.1093/ntr/ntw080

37. Balmford J, Borland R, Hammond D, et al. Adherence to and reasons for premature discontinuation from stop-smoking medications: data from the ITC Four-Country Survey. Nicotine Tob Res. 2011;13 (2):94-102. doi:10.1093/ntr/ntq215

38. Hood NE, Ferketich AK, Paskett ED, et al. Treatment adherence in a lay health adviser intervention to treat tobacco dependence. Health Educ Res. 2013;28(1):72-82. doi:10.1093/her/cys081

39. Lewis JA, Senft N, Chen H, et al. Evidence-based smoking cessation treatment: a comparison by healthcare system. BMC Health Serv Res. 2021;21(1):33. doi:10.1186/s12913-020-06016-5

40. Yingst JM, Veldheer S, Hrabovsky S, et al. Reasons for non-adherence to nicotine patch therapy during the first month of a quit attempt. Int $J$ Clin Pract. 2015;69(8):883-888. doi:10.1111/ ijcp. 12644

41. Pezzuto A, Lionetto L, Ricci A, et al. Inter-individual variation in CYP2A6 activity and chronic obstructive pulmonary disease in smokers: perspectives for an early predictive marker. Biochim Biophys Acta Mol Basis Dis. 2021;1867(1):165990. doi:10.1016/j. bbadis. 2020.165990

\section{Publish your work in this journal}

The International Journal of COPD is an international, peer-reviewed journal of therapeutics and pharmacology focusing on concise rapid reporting of clinical studies and reviews in COPD. Special focus is given to the pathophysiological processes underlying the disease, intervention programs, patient focused education, and self management protocols. This journal is indexed on PubMed Central, MedLine and CAS. The manuscript management system is completely online and includes a very quick and fair peer-review system, which is all easy to use. Visit http://www.dovepress.com/testimonials.php to read real quotes from published authors. 